\title{
SPATIAL THEORY AND HUMAN BEHAVIOR
}

\author{
GUNNAR OLSSON and STEPHEN GALE, University of Michigan
}

A significant number of spatial analysts have recently shifted their emphasis away from economic theories of location to a more behavioristic approach. It is the purpose of this paper to review this development and to suggest a general framework within which an integrated theory of human spatial behavior can be developed. More specifically, the first part of the paper will isolate the major behavioristic and spatial assumptions in classical location theory; the second section will deal with some recent attempts to relax the most limiting of these assumptions. Since a large part of this new work has employed probability models, some comments will be directed toward the concept of stochastic model building in general. In the last part of the paper, this discussion will be extended to comments on $q$-way probability matrices and their use in multidimensional Markovian models.

\section{ECONOMICS AND SPATIAL THEORY}

It has been argued that the basic problem in economics is to study how limited material resources become allocated among different users. This allocation process can be viewed as a game between producers and consumers in which economic man behaves in a predictable manner. More precisely, the consumer is assumed to follow a strategy that maximizes his utility function, while the producer attempts to maximize his profit. In this simplified economic game, both players operate within given constraints, such as budget, demand, technology, etc., and each is further restrained by the moves of his opponent. After a period of mutual adjustment, both will find that they cannot improve their respective positions and an equilibrium situation has been reached (38). The allocation problem can be analyzed with tools other than game theory and equilibrium models, but most available techniques are in fact based on the same premises. This indicates that Katona's and Simon's characterization of classical economics is still basically valid and that most existing theories are normative $(36,58)$.

Since most spatial theories are extensions of economic theories, traditional work in regional science and theoretical geography has been directed toward the same normative, optimizing constructs as have the studies in economics. The modifications of classical economics by Lösch, Isard, and others have led to a relaxation of the presumption of a nondimensional economic world but they have not affected any of the normative-behavioristic assumptions. As a consequence, the players in the spatial economic game are blessed with the attributes of economic man, which makes it meaningful to apply the same equilibrium approach as in nonspatial economics. The extension of the classical theory into edged.

The support of the Swedish Council for Social Science Research is gratefully acknowl- 
a spatial context implies, however, that the players can influence the outcome of the game not only by manipulating utility and production functions, or supply and demand curves, but also by manipulating space. In a ceteris paribus situation, this indicates that the producer tries to increase his profits by enlarging the size of his market area, while the consumer tries to minimize his purchasing costs by forcing the producer to come as close to him as possible.

It was in this areal sense that von Thünen and Lösch treated space in their models. Lösch arrived at his spatial solution by combining the notion of the demand cone with a set of special areal equilibrium conditions which stated that no parts of the total area can be left unserved, and that supply, production, and sales areas must be as small as possible (40). Isard later extended this into a more general formulation embracing both Lösch's and von Thünen's derivations as special cases (31). This was made possible by the use of a substitution framework in which the notion of transportation inputs played a major role. As a consequence, Isard was able not only to fuse existing location theories into one model but also to incorporate pertinent parts of production and distribution theory.

In addition to the studies of space in the areal sense, there exists a large number of quasi-spatial models in which space has been simplified and collapsed into a set of points. Some interregional equilibrium models are good examples of this approach (34) as are the transportation variant of the linear programming model, most gravity formulations, and the application of graph theory to spatial problems (47). In other cases, such as general interregional linear programming and input-output analysis, space has been included in the form of point regions simply by expanding the traditional models (32); symbolically, this means that new subscripts and superscripts have been introduced and the traditional equations have been repeated for each region.

\section{Assumptions}

It has already been noted that most spatial theories are based on the same behavioristic assumptions as the nondimensional theory of the firm. Cyert and March (14, p. $8 \mathrm{ff}$.) have suggested that these premises can be reduced to two rationality assumptions; first, that firms consistently seek to maximize profits and, second, that they possess perfect knowledge. It is only if these assumptions are fulfilled that traditional spatial theory is valid, and it is only then that it is meaningful to employ it in the computation of optimality and equilibrium solutions.

In spatial theories, the maximization of profits has usually been assumed to result from the minimization of transportation costs or, in the simplified case, from the minimization of physical distance. In this respect, locational optimality can be related to the social physics principle of least effort, which Bunge recently restated as a nearness principle by which he means that interacting objects tend to place themselves as closely together as possible (4). In effect, Bunge and the social physicists have drawn analogies with the fundamental optimality principles in physics, and, in this respect, their approach is similar to some biological studies (56) and to general systems theory in particular (1). 
It is superfluous to discuss in any detail how the goal of maximizing profits has been criticized in economics. It is enough to recall that particular attention has been drawn to the importance of noneconomic motives in the decision-making process of the firm. As a result, it has often been suggested that the optimizing function should include noneconomic factors and that the objective, or purely economic, maximizing function be replaced by a subjective one. For the spatial analyst, similar reasoning implies that minimization of physical distance is only a theoretical notion intended to simplify the formulation of abstract concepts. Actors in the real world realize of course that minimizing functional or subjective distance is often more appropriate than putting interacting objects as closely together as possible in the physical sense. The use of subjective distance functions in spatial models is closely related to the use of subjective utility and profit functions in nondimensional economics, and, in this regard, subjective distance could be one of the variables entered into those functions. A seemingly nonoptimal location decision or a biased interaction field, for example, may appear optimal for the individual concerned simply because he understands how to specify the relevant subjective distance and maximizing functions.

The notion of subjective distance functions is also relevant to an understanding of Cyert and March's second proposition, i. e,, that the classical rationality assumption presupposes perfect knowledge on the part of the decision makers. What appears as an optimal location or interaction pattern for the individual may thus do so simply because he is not aware of all the possibilities. For the most part, this unawareness relates either to insufficient information channels or to the decision-maker's subjective filtering of the information he receives. This suggests a close relationship between interaction fields and information flows of the type discussed in several migration studies $(29,30,41,46,63)$. Pred has treated urban and industrial growth in similar terms and suggested that spatial economic growth is governed by a circular and cumulative process by which the creation and dissemination of inventions is facilitated (52). Such processes create tendencies toward selected growth, and these are further enhanced by biases in the flow of information. Thorngren (59) and Wärneryd (65) have analyzed the Swedish economy in related terms and found that the mechanisms of spatial growth are best understood if existing spatial theories are complemented with the concepts of organization theory. Pred (53) has recently extended his ideas on circular and cumulative causation into a detailed and excellent discussion of the behavioristic assumptions in location theory-reemphasizing the fact that spatial growth, to a large extent, reflects the spatial patterns of information flows.

As already noted, the data submitted through biased information channels are further distorted by each individual's perception or subjective filtering of the information he receives. On a large scale, Gould (23) has demonstrated that such distortions of information content can influence people's ranking of different countries, and Wolpert (64) has noted how the perception of a social and physical environment affects an individual's decision to migrate. Finally, a number of related studies has treated the perception of natural hazards as a particular aspect of the physical environment $(5,35,39,57,62)$. 
These brief comments on the perception and flow of information indicate how the subjective evaluation of spatial alternatives makes the traditional behavioristic assumptions extremely unrealistic. In addition, spatial theories and models often involve specifically spatial premises that are equally questionable. Thus, most areal spatial models depend on the notion of a homogeneous plain over which the actors in the economic game move themselves and their activities. In the case of the interregional point models, on the other hand, it is not always clear which spatial assumptions have been made; presumably, however, they all involve some measure of areal dispersion and homogeneity which is then used as a basis for determining the center of each region. If this is done on a functional basis, each region may be imagined as a punctiformally generated demand cone of the Lösch-type or as a Clark-type city (7) in which sales, interaction intensities, and population densities decrease with increasing distance from the center. Gurevich and Saushkin (24) have discussed this problem and have demonstrated that Clark's model can be extended to take nonhomogeneities into account. Their contribution is important since reality is only remotely similar to the theoretical isotropic plain - a recognition that has caused many empirical geographers to refute practically all spatial theory. One of the few constructive suggestions in this dialogue between empricists and theoreticians has been provided by Tobler (60) in his work on mathematical functions by means of which complex realities can be transformed into homogeneous plains. Conceptually, his approach is related to that of von Thünen, who deformed the theoretical pattern of concentric land-use zones by introducing a river on which goods could be transported more cheaply than over the land.

\section{Recent Approaches to Spatial Model Building}

The discussion thus far has recapitulated and criticized the basic assumptions in classical spatial theories. There is general agreement that these assumptions are highly unrealistic, but there has been little work on the specification of another more rational set of premises. Furthermore, the few attempts along these these lines have been primarily verbal rather than symbolic and critical rather than constructive. One reason for this may be that knowledge about spatial behavior is far from adequate. Isard and Dacey (33) came to similar conclusions in their study of individual behavior in which they pointed out that an individual's choice among alternatives varies widely with his attitudes. By restating the assumptions of rational behavior in axiomatic form they were able to isolate the few instances in which spatial behavior can comply with theoretical expectations. In situations where the knowledge is so scant, it is meaningless to employ highly simplified deterministic models with predefined cause and effect relationships. Some economists have therefore suggested that the classical homo economicus be replaced by a homo stochasticus, and it is obvious that a concomitant modification of spatial theory is highly desirable. As noted by Curry (12), in a discussion of spatial probability models, an important advantage of the probabilistic approach is precisely this explicit admission of the researcher's ignorance. 
Although there are great conceptual differences between deterministic and stochastic approaches, it often happens that the outcomes from the two types of formulations are the same. As a consequence, the insights into a process provided by one model can help in the interpretation and development of another logically more attractive one. For the spatial analyst, it is particularly fortunate that this applies to one of his most widely used models. The basically deterministic gravity formulation can thus be related to several existing stochastic models of human interaction, and it can, in fact, itself be transformed into a model of that type (50). Typically, these interaction models consider two sets of probabilities, one set specifying the number of opportunities already reached and accepted by a trip maker, and the second set specifying the proportion of trip makers going beyond a certain distance to reach an acceptable opportunity. Consequently, the probabilities themselves relate human behavior to the spatial unevenness of opportunities and therefore indirectly to the spatial homogeneity assumption.

The probabilities in some cell counting models can be interpreted in essentially the same manner. Medvedkov (45), for example, has employed the entropy concept of information theory as a technique for quantifying the agreement between empirical and theoretical settlement distributions. More specifically, he suggested that Christaller's hexagonal central place pattern be regarded as the signal, and the random noise be attributed to deviations from the isotropic conditions. Medvedkov thus viewed the spatial distribution of places as a result of a set of "regulated accidents" in which chance or randomness has affected the outcome from the otherwise deterministic central place theory. A similar approach has been taken by Dacey $(15,16,17,18,19)$ who has conceived of urban places as points which topography or institutional factors have dislocated from their theoretical positions on the hexagonal lattice. A variant of the cell counting technique was used to verify that the distribution of places in almost homogeneous areas follows a double Poisson distribution, although the locations in inhomogeneous areas are better described by a double negative binomial. These and other distribution functions have recently been discussed by Olsson (49). Hudson (28) has used them in his analysis of rural settlement and Rogers (55) has employed them in studies of shops within cities. Finally, Harvey (25) has furnished an excellent discussion of cell count analysis in diffusion studies, pointing out, in particular, how the technique offers a way in which time and space can be treated in the same model.

Even though the relationships between distribution functions and physical urn models make it possible to draw conclusions about generating processes, the great value of cell counting analysis is as a sophisticated technique for spatial description. Implicit in this approach to description is the idea that the real world represents only one of many possible realizations of a specified stochastic process. If this realization has had a small likelihood of occurrence, it in itself becomes difficult to understand without reference to the underlying probability matrix from which chance happened to pick an unusually small figure. What may initially appear to be a unique case can thus be fitted into more general constructs. This demonstrates that even basically descriptive probability models lend themselves to causal inference by which large scale aggregate regularities 
can be related to small scale processes. Employing a stochastic approach, one may therefore determine the degree to which reality deviates from the homogeneous plain and also, in theoretical terms, account for this deviation. If the focus is on the behavior of real world people, as compared to the behavior assumed in traditional spatial theory, the possibilities for such causal inferences become indispensable.

An interesting and somewhat different attempt along these lines has been made by Curry (9), who claims that the time factor is the key to understanding the spatial distribution of central place activities. In particular, he has suggested that the spatial pattern of service outlets is a result of consumer behavior and its changes over time. Employing a set of Poisson distributions with the mean varying between different goods, Curry used this approach to determine not only the number of purchases per week but also the size of various service areas. Most importantly, he later extended these arguments into a general theory of consumer and retailer behavior (13) in which interesting analogies were drawn from turbulence and communications theories. As a consequence, the use of spectral analysis was suggested as an appropriate technique both for determining the convertability between the time and space domains and for detecting noise due to uncertainties faced by consumers and suppliers.

Spectral analysis offers a new and challenging approach to traditional spatial theory, primarily because it provides a sophisticated technique for describing and analyzing an agglomerated spatial economy in terms of its harmonics. The generation of such an economy can be related to the flow of information as Pred has proposed $(52,53)$, but it may also be understood in terms of the stochastic interplay between the forces of distance and specialization as suggested by Curry (11). More specifically, the generation of an undulating spatial economic surface can be related to the idea of contingency in probability theory; this, by definition, means that one particular outcome of a stochastic process can change the probability for similar events occurring in spatial and temporal proximity. In one of Curry's examples (11), such an accumulating process gave rise to high industrial and areal specialization as in the American manufacturing belt; in another case (10), it provided an explanation for the occurrence of ice ages.

\section{SUGGESTED FRAMEWORK FOR SPATIAL BEHAVIORAL ANALYSIS}

The models reviewed in the preceding section are all promising and attractive, even though Curry's explicit recognition of chance and uncertainty makes his approach more appealing than most of the others. No matter how thought-provoking a particular formulation may be, however, it is always worth recalling that several quite different models sometimes give rise to almost identical results (6). Attention will therefore be drawn to a somewhat different, but very general framework within which spatial theory and human behavior may be studied.

Keeping in mind that precise knowledge about spatial behavior is lacking, a deterministic approach seems indiscriminate. Further, recalling that human behavior is affected by a large number of variables, it appears appropriate to consider explicitly the complexity or multidimensionality of the underlying data. 
Finally, a model of behavioral processes should treat spatial and temporal sequences concomitantly.

\section{Traditional Markov Models}

If these are the three central criteria which the ideal model of spatial behavior must meet, then it is possible that a Markovian formulation can be extended to provide a meaningful analytic framework. To facilitate a more detailed elaboration of this point the basic properties of Markovian models will be briefly summarized.

The general Markov process $(2,37,51)$ is a sequential model characterized by a set of state conditions, $S$, where

$$
S=\left\{s_{1}, \ldots, s_{m}, \ldots\right\}
$$

and by a set of sequence conditions, $T$, where

$$
T=\left\{t_{1}, \ldots, t_{n}, \ldots\right\} \text {. }
$$

In addition, the Markov process embodies a probability criterion, termed the Markov property, defined as

$$
P\left(t_{n}=s_{i} \mid t_{n-1}, \ldots, t_{n-j}=s\right)=P\left(t_{n}=s_{1} \mid t_{n-1}=s_{j}\right)
$$

which says that the probability that any state $s_{i}$ occurs on sequence $t_{n}$ is independent of all other states in the sequence except that one which occurred on $t_{n-1}$. In operational terms, the Markov process is a sequence of matrix operations of the form

$$
{ }^{n} P \cdot{ }^{n} V={ }^{n+1} V^{\prime}
$$

where $P$ is a matrix of transition probabilities, and $V$ is a vector of observations. The superscript in (4) represents the element $t_{n}$ from the set of sequences $T$, where time is the implicit variable in the model. The cells of the matrix $P$, $\left\{p_{i j}\right\}$, are the probabilities of moving from state $i$ to state $j$. Functionally the $\left\{p_{i j}\right\}$ can be denoted as

$$
{ }^{n+1} p_{i j}=f\left({ }^{n} p_{i j},{ }^{n} v_{j}, n\right)
$$

which means that the probability matrix in a Markov process is related to the value of the probability matrix at the preceding sequence, the observed state $v_{j}$ at the preceding sequence, and the place of that particular sequence in the set $T$. Given a process in a particular state $s_{i}$ at sequence $t_{n}$, no additional information is therefore needed to characterize completely the distribution of the process at any time $t>t_{n}$.

Whereas the general class of Markov process models have not found wide application in the study of social processes, Markov chains have been used to a considerable extent. Since the Markov chain forms a subset of the Markov process, it is characterized by the same basic properties; the only difference is that a Markov chain assumes that the probability that any state $s_{i}$ occurs on a particular sequence $t$ is independent of the position of that sequence in the set $T$. The condition for a Markov chain can therefore be written as 


$$
P\left(t_{n}=s_{i} \mid t_{n-1}, \ldots, t_{n-j}=s\right)=P\left(t_{m}=s_{i} \mid t_{m-1}, \ldots, t_{m-j}=s\right)
$$

which implies that a Markov chain is a Markov process with the added condition of stationarity. Operationally, this means that the matrix $P$ is invariant over time such that

$$
{ }^{n} P_{i j}={ }^{0} P_{i j}
$$

where ${ }^{0} P_{i j}$ represents the initial value of the transition probability matrix at $t_{0}$. Since each combination of $V$ and $P$ thus results in a unique Markov chain, simple matrix operations can be used to determine the steady state or equilibrium conditions for the model.

There exists a number of applications of Markovian models to spatial studies. Brown (3), for instance, employed a chain model to describe the spread of liquid propane tanks, and Rogers (55) used a similar formulation in his analysis of interregional migration flows. However, migration behavior does not usually conform to the stationarity assumption in the Markov chain model, and some writers have consequently suggested that Markov process models be used instead $(42,43)$. As the transition probability matrices in the latter can change over time, they are well suited for handling problems involving search procedures and learning processes of the type encountered not only in migration but also in marketing behavior $(22,26)$. In addition, Markovian models have been applied in a number of other spatial studies $(8,21,27,44)$ with subjects ranging from city size distributions to the movement of points in two dimensions.

\section{A Variation}

The brief summary of Markovian models and their use in spatial research indicates at least three points worthy of further attention. Firstly, the traditional models employ ordinary matrix structures which limit the number of analyzed variables to one. Secondly, the ordinary rules for matrix multiplication imply a linearity assumption such that the element $j$ in ${ }^{n+1} V$ in (4) is equally dependent on all of the entries of the $i^{\text {th }}$ row of ${ }^{n} P$ and the $j^{t h}$ column of ${ }^{n} V$. Thirdly, the traditional model is limited in its temporal scope because it specifies the probability of any state $s_{i}$ occurring on sequence $t_{n}$ to be independent of all states in the sequence except the immediately preceding one.

It is almost a truism that models are used in scientific investigations because they limit the number of variables employed in the analysis. With respect to spatial behavior, however, present knowledge is too scant to suggest which of the many possible variables should be employed. To limit oneself to the use of the one (or sometimes two) variables whose interactions can be portrayed in ordinary matrix structures would therefore imply severely limiting ceteris paribus assumptions. The answer to this problem comes in a logical extension of the matrix form such that the ordinary two-dimensional matrix

$$
P=\begin{array}{ccc}
p_{11} & \cdots & p_{1 m_{2}} \\
\vdots & & \vdots \\
p_{m_{1} 1} & \cdots & p_{m_{1} m_{2}}
\end{array}
$$


be expanded into three dimensions, where

$$
P^{\prime}=\begin{array}{cccc} 
& p_{11 m_{3}} & \cdots & p_{1 m_{2} m_{3}} \\
p_{111} & \cdots & \cdots & p_{1 m_{21}} \\
\vdots & \vdots & \vdots & \vdots \\
\vdots & p_{m_{1} m_{3}} & \cdots & \vdots \\
\vdots & p_{m_{1} m_{2} m_{3}} \\
p_{m_{1} 11} & \cdots \cdots & \cdots & p_{m_{1} m_{2}}
\end{array}
$$

or, in the more general case, into a matrix of $q$ dimensions. Following Oldenburger's notation (48), such a structure can be called a $q$-way matrix represented as

$$
P^{\prime \prime}=\left\{p_{m_{1}}, m_{2}, \ldots, m_{m_{q}}\right\},
$$

i.e., as a vector space with any number of axes, $q$, where $q$ is a finite positive integer.

To appreciate the significance of $q$-way matrices, it may be helpful to recall how Clark (8) tested the usefulness of Markov chain models as a tool for analyzing rental housing distributions in urban areas. As noted by Clark himself, the limitations of the two-dimensional transition probability matrix allowed him to trace only the movement of a tract within the rental classes; the spatial pattern of these movements could not be considered explicity. This means that the traditional Markov chain approach enabled him to derive a gross measure of change in one variable at the expense of neglecting the associated spatial process. Map analysis could shed light on this problem, but it could be treated more directly by means of a four-way matrix, allowing the indexing not only of the probabilities of moving within rental classes but also within space.

To turn to the second point raised above-i.e., the operator in Markov models-it should be recalled that ordinary matrix multiplication can be functionally denoted as

$$
V^{\prime}=f(V, P)
$$

where $f$ is a linear transformation specified as

$$
v_{i j}^{\prime}=\sum_{k} p_{i k} \cdot v_{i k j} \text {. }
$$

Historically, this operator was derived for the solution of sets of simultaneous linear equations, but its inherent property of linearity could become a limiting factor when applied to models of social processes. As seen from (9) and (10), the function $f$ is solely responsible for the linearity of the transformation; the matrices $V, P, V^{\prime}$ serve merely as indexing or representational mechanisms. The obvious generalization of the sequential operator therefore involves a redefinition of $f$ into a function, $f^{\prime}$, which can be either linear or nonlinear, continuous or discontinuous.

Much work has yet to be done on the specification of the function $f^{\prime}$, but a similar approach has recently been used by Tobler (61), who suggested a twoway linear transformational model of the form

$$
V^{\prime}=P \cdot V \cdot P^{T}
$$


where $P^{T}$ is the transpose of the transition probability matrix $P$. Since the multiplication in Tobler's case still is defined as a linear operator, the premultiplication of $V$ by $P$ and the postmultiplication of $V$ by $P^{T}$ operates respectively on the rows and the columns of $V$. Thus $V^{\prime}$ is a linear combination of both the associated rows and columns of the probability matrix, such that

$$
\begin{aligned}
& v^{\prime \prime}{ }_{i j}=\sum_{k} p_{i k} v_{k j} \\
& v_{i j}^{\prime}=\sum v^{\prime \prime}{ }_{i k} p_{k j}^{T}
\end{aligned}
$$

where $V^{\prime \prime}$ is employed solely for computational simplicity. Tobler has also discussed the relationship between (12) and the idea of a local operator by which the interaction between juxtaposed elements can be established. For the twoway matrix, the local operator can be defined as

$$
\begin{aligned}
v_{i j}^{\prime}= & f\left[\left(v_{i-1, j-1}, v_{i-1, j}, v_{i-1, j+1}, v_{i, j-1}, v_{i, j}, v_{i, j+1}, v_{i+1, j-1}, v_{i+1, j},\right.\right. \\
& \left.v_{i+1, j+1}\right),\left(p_{i-1, j-1}, p_{i-1, j}, p_{j-1, j+1}, p_{i, j-1}, p_{i, j}, p_{i, j+1},\right. \\
& \left.\left.p_{i+1, j-1}, p_{i+1, j}, p_{i+1, j+1}\right)\right]
\end{aligned}
$$

from which generalization to the case of $q$-way matrices follows immediately.

The concept of a local operator is closely related to the notion of neighborhood effects in spatial interaction and diffusion. It seems possible, therefore, that detailed knowledge of systematic variations in the $b$-value of the gravity model may help to determine precisely multiplication rules that would yield the desired spatial contingency effects. Presently, it is less clear how the operator should be defined for those variables having no spatial dimension.

Finally, to turn to the third point raised above, it should be recalled that the transition probability matrices are usually determined from the formulation

$$
P=\left[p_{i j}\right]=\frac{a_{i j}}{\sum_{j} a_{i j}}
$$

where the $\left\{a_{i j}\right\}$ are the observed frequencies of transitions from state $i$ to state $j$. The results of this calculation apply to ordinary matrix structures, but, once again, they can easily be extended to $q$-way matrices, although the interpretation of each cell of the probability matrix is then altered; in the two-way matrix, each $p_{i j}$ represents the many-to-one mapping of a complex process, while the values in the $q$-way matrix specify simple conditional probabilities. In both cases, however, the probability statements are empirical and consequently conceived of in an empirical or frequentist manner. In the case of behavioral modeling, such a conception could be somewhat limiting and it may be more attractive to employ the subjectivist view instead.

The specification of the matrix $P$ also bears on another problem related to Markov models. Thus, the Markov property states that the outcome of each trial depends solely on the outcome of the immediately preceding one which makes the length of each trial period crucial. If it is desirable to keep the Markov property unchanged, the obvious solution to this difficulty is to determine the probabilities over short time periods. If, on the other hand, it is deemed more meaningful to change the Markov property itself, the transition probabil- 
ities can be made functions of any number of previous states, or symbolically

$$
P\left(t_{n}=s_{i} \mid t_{n-1}, \ldots, t_{n-j}=s\right)=P\left(t_{n}=s_{i} \mid t_{n-1}, \ldots, t_{n-a}=s^{\prime}\right)
$$

where $\mathrm{a}$ is any positive finite integer $\leq n$.

\section{SUMMARY AND CONCLUSIONS}

The first part of this paper discussed the well-known relationships between classical economics and spatial theory, and it was noted that most spatially oriented studies have been based on the same behavioristic assumptions as the nondimensional theory of the firm. To overcome the limitations imposed by these assumptions, it was pointed out that subjective distance functions must be introduced as a complement to the economists' subjective profit maximizing functions.

After this introduction, the paper turned to a discussion of the notion of subjective functions and the manner in which they might be entered into operational spatial models. Since the knowledge of spatial behavior is limited, it was argued that the use of simplified deterministic models with predefined cause and effect relationships would be meaningless. Therefore, as a preliminary to the search for more appropriate constructs, the use of probabilistic models in spatial research was reviewed. Particular attention was drawn to Curry's studies, in which stochastic process models have been employed as a device for relating observed large-scale regularities to small-scale behavior.

Although much still remains to be done, most of the models discussed in the second part are promising. Recalling, however, that two very different formulations sometimes may produce the same outcome, the third part of the paper draws attention to an alternative approach. More specifically, it was suggested that the usefulness of Markovian models in spatial research should be investigated in more detail, even though a subsequent review of existing studies indicated that traditional Markov formulations may impose several limitations. As an example, it was noted that the use of ordinary two-way matrix structures in effect limits the number of variables to one necessitating far reaching ceteris paribus assumptions. It was suggested that the easiest way to avoid this difficulty was to extend the ordinary matrices into several dimensions. This, however, raised the question of how to define appropriate multiplication rules, and it was noted that the operator can be specified in a large number of ways from which the researcher can then choose those most relevant to his specific problem. With respect to spatial studies, it was argued that the condition of a linear sequential operator be modified in order to account for neighborhood and contingency effects.

The suggestions concerning $q$-way matrices and local operators are admittedly very general, and much work remains before they can be made operational and subsequently employed in testable models. In this respect, the discussion has been typical of the inductive stage in research. Experiments with a set of specific models of the optimality type have thus indicated the respects in which spatial theories are least satisfactory, but these experiments have not shown how existing models can be reformulated to take the noted deficiencies into ac- 
count. In such situations, it is often necessary to sacrifice simplicity for accuracy and increase the number of variables until the mechanisms of the studied process are fully understood. Once this understanding has been reached, the number of variables may then be reduced to achieve the economy of thought which is a primary characteristic of a good model. It is possible that the notion of $q$-way matrices and multidimensional Markov formulations constitute a framework for the analysis of spatial behavior in general and for the derivation of simplified but realistic models in particular.

\section{REFERENCES}

1. von Bertalanffy, Ludwig. "General System Theory: A Critical Review," General Systems, VII (1962), pp. 1-20.

2. Bharucha-Reid, A. T. Elements of the Theory of Markov Processes and Their Applications. New York City: McGraw-Hill Book Company, Inc., 1960.

3. Brown, Lawrence A. "The Diffusion of Innovation: A Markov Chain-Type Approach." Northwestern University: Department of Geography, Discussion Paper No. 8 (1963).

4. Bunge, William. Theoretical Geography. Lund: Gleerups Förlag, 1966.

5. Burton, Ian and Kates, Robert W. "Perception of Natural Hazards in Resources Management," Natural Resources Journal, III (1964), pp. 412-41.

6. Chorley, Richard J. "Geography and Analogue Theory," Annals of the Association of American Geographers, LIV (1964), pp. 127-37.

7. Clark, Colin. "Urban Population Densities," Journal of the Royal Statistical Society, Series A, CXIV pp. 490-96.

8. Clark, W. A. V. "Markov Chain Analysis in Geography: An Application to the Movement of Rental Housing Areas," Annals of the Association of American Geographers, LV (1965), pp. 351-59.

9. Curry, Leslie "The Geography of Service Centers within Towns: The Elements of an Operational Approach" in Knut Norborg ed., Proceedings of the IGU Symposium in Urban Geography, Lund 1960. Lund: Gleerups Förlag, 1962.

10. - "Climatic Change as a Random Series," Annals of the Association of American Geographers, LII (1962), pp. 21-31.

11. W. "The Random Economy: An Exploration in Settlement Theory," Annals of the Association of American Geographers, LIV (1964), pp. 138-46.

12. "Chance and Landscape" in J. W. House ed., Northern Geographical Essays in Honour of G.W.J. Daysh. Newcastle-upon-Tyne University: Department of Geography Publications (1967).

13. - - "Central Places in the Random Spatial Economy," Journal of Regional Science, VII, No. 2 (Supplement) (1967), pp. 217-38.

14. Cyert, Richard M. and March, James G. A Behavioral Theory of the Firm. Englewood Cliffs, New Jersey: Prentice Hall, Inc., 1963.

15. Dacey, Michael F. "Modified Probability Law for Point Patterns More Regular than Random," Annals of the Association of American Geographers, LIV (1964), pp. 559-65.

16. —_ "Order Distance in an Inhomogeneous Random Point Pattern," Canadian Geographer, IX (1965), pp. 144-53.

17. - "A Probability Model for Central Place Locations," Annals of the Association of American Geographers, LVI (1966), pp. 549-68.

18. "A Compound Probability Law for a Pattern More Dispersed than Random and with Areal Inhomogeneity," Economic Geography, XLII (1966), pp. 172-79. 
19. - "A County Seat Model for the Areal Pattern of an Urban System," Geographical Review, LVI (1966), pp. 527-42.

20. Feller, William. An Introduction to Probability Theory and Its Applications, Volume I. New York City: John Wiley \& Sons, Inc., 1957.

21. Fuguitt, Glenn W. "The Growth and Decline of Small Towns as a Probability Process," American Sociological Review, XXX (1965), pp. 403-11.

22. Golledge, Reginald G. "A Conceptual Framework of a Market Decision Process," University of Iowa: Department of Geography, Discussion Paper No. 4 (1967).

23. Gould, Peter R. "On Mental Maps," Michigan Inter-University Community of Mathematical Geographers, Discussion Paper No. 9, (1966).

24. Gurevich, B. L. and Sauskin, Yu G. "The Mathematical Method in Geography," Soviet Geography, VII, No. 4 (1966), pp. 3-35.

25. Harvey, David. "Geographic Processes and the Analysis of Point Patterns: Testing Models of Diffusion by Quadrat Sampling," Transactions of the Institute of British Geographers, XL (1966), pp. 81-95.

26. Herniter, Jerome D. and Howard, Ronald A. "Stochastic Marketing Models" in David B. Hertz et al. eds., Progress in Operations Research, Volume 2. New York City: John Wiley \& Sons, Inc., 1964.

27. Hudson, John C. Maps and Spatial Processes Describable with Markov Chains. University of North Dakota: Department of Geography, mimeograph (1966).

28. - Theoretical Settlement Location. Unpublished doctoral dissertation, Department of Geography, University of Iowa, 1967.

29. Hägerstrand, Torsten. Innovationsforloppet ur korologisk synpunkt. Lund: Gleerups Förlag, 1953.

30. - "Migration and Area" in David Hannerberg et al. eds., Migration in Sweden. Lund: Gleerups Förlag, 1957.

31. Isard, W/alter. Location and Space Economy. New York City: John Wiley \& Sons, Inc., 1956.

32. - Methods of Regional Analysis: An Introduction to Regional Science. New York City: John Wiley \& Sons, Inc., 1960.

33. Isard, Walter and Dacey, Michael F. "On the Projection of Individual Behavior in Regional Analysis, I and II," Journal of Regional Science, IV (1962), pp. 51-96.

34. Isard, Walter and Ostroff, David. "General Interregional Equilibrium," Journal of Regional Seience, II (1960), pp. 67-74.

35. Kates, Robert W. Hazard and Choice Perception in Flood Plain Management. University of Chicago: Department of Geography, Research Paper No. 78, (1962).

36. Katona, George. Psychological Analysis of Economic Behavior. New York City: McGraw-Hill Book Company, Inc., 1951.

37. Kemeny, John G. and SnelI, J. Laurie. Finite Markov Chains. Princeton, New Jersey: D. Van Nostrand Company, Inc., 1960.

38. Kuenne, Robert E. The Theory of General Economic Equilibrium. Princeton, New Jersey: Princeton University Press, 1963.

39. Lowenthal, David ed. Environmental Perception and Behavior. University of Chicago: Department of Geography, Research Paper No. 109 (1967).

40. Lösch, August. The Economics of Location, Woglom-Stolper translation. New Haven, Connecticut: Yale University Press, 1954.

41. Lövgren, Esse. "The Geographical Mobility of Labor," Geografiska Annaler, XXXVIII (1956), pp. 344-94.

42. McGinnis, Robert, Myers, George C., and Pilger, John E. Internal Migration as a Stochastic Process. Paper presented at the meeting of the International Statistical Institute, Ottawa, August, 1963. 
43. McGinnis, Robert and Pilger, John E. On a Model for Temporal Analysis, Paper presented at the meeting of the American Sociological Association, Los Angeles, August, 1963.

44. Marble, Duane F. “Simple Markovian Model of Trip Structure in a Metropolitan Region," Proceedings of the Western Section of the Regional Science Association, 1964.

45. Medvedkov, Yuriy V. "The Regular Component in Settlement Patterns Shown on a Map," Soviet Geography, VIII (1967), pp. 150-68.

46. Nelson, Phillip. "Migration, Real Income and Information," Journal of Regional Science, I (1959), pp. 43-74.

47. Nystuen, John D. and Dacey, Michael F. "A Graph Theory Interpretation of Nodal Regions," Papers and Proceedings of the Regional Science Association, VII (1961), pp. $29-42$.

48. Oldenburger, R. "Composition and Rank of $n$-way Matrices and Multilinear Forms," Annals of Mathematics, XXV, No. 3 (July, 1934), pp. 622-57.

49. Olsson, Gunnar. "Lokaliseringsteori och stokastiska processer," in Tor Fr. Rasmussen ed., Forelesninger $i$ regionale analysematoder. Oslo: Norsk Institutt for By-og Regionforskning, 1967.

50. ㄴ "Central Place Theory, Spatial Interaction, and Stochastic Processes," $P a$ pers of the Regional Science Association, XVIII (1967), pp. 13-45.

51. Parzen, Emanuel. Stochastic Processes. San Francisco: Holden-Day, 1962.

52. Pred, Allan. The Spatial Dynamics of Urban-Industrial Growth 1800-1914: Interpre. tive and Theoretical Essays. Cambridge, Massachusetts: The M.I.T. Press, 1966.

53. - Behavior and Location. Foundations for a Geographic and Dynamic Location Theory, Part I. Lund: Gleerups Förlag, 1967.

54. Rogers, Andrei "A Stochastic Analysis of the Spatial Clustering of Retail Establishments," Journal of the American Statistical Association, LX (1965), pp. 1094-1103.

55. _A Markovian Policy Model of Interregional Migration," Papers of the Regional Science Association, XVII (1966), pp. 205-24.

56. Rosen, Robert. Optimality Principles in Biology. London: Butterworths, 1967.

57. Saarinen, Thomas F. Perception of the Drought Hazard on the Great Plains. University of Chicago: Department of Geography, Research Paper No. 106 (1966).

58. Simon, Herbert A. Models of Man. New York City: John Wiley \& Sons, Inc., 1957.

59. Thorngren, Bertil. Regional External Economies. Stockholm School of Economics: The Economic Research Institute, mimeograph (1967).

60. Tobler, Waldo R. "Geographic Data and Map Projections," Geographical Review, LIII (1963), pp. 59-78.

61. ․ "Of Maps and Matrices," Journal of Regional Science, VII, No. 2 (Supplement) (1967), pp. 275-80.

62. White, Gilbert F. Choice of Adjustment to Floods. University of Chicago: Department of Geography, Research Paper No. 93 (1964).

63. Wolpert, Julian. "Bt:avioral Aspects of the Decision to Migrate," Papers of the Regional Science Association, XV (1965), pp. 159-69.

64. - "Migration as an Adjustment to Environmental Stress," Journal of Social Issues, XXII, No. 4 (1966), pp. 92-102.

65. Wärneryd, Olof. "Urban Regions as Spatial Systems." Paper presented at the second Polish-Scandinavian Regional Science Meeting, Copenhagen, 1967. 